\title{
LATTICE SUBGROUPS OF FREE CONGRUENCE GROUPS
}

\author{
by A. W. MASON \\ (Received 16 February, 1968)
}

1. Introduction. Let $\Gamma(1)$ denote the homogeneous modular group of $2 \times 2$ matrices with integral entries and determinant 1 . Let $\hat{\Gamma}(1)$ be the inhomogeneous modular group of $2 \times 2$ integral matrices of determinant 1 in which a matrix is identified with its negative. $\hat{\Gamma}(N)$, the principal congruence subgroup of level $N$, is the subgroup of $\hat{\Gamma}(1)$ consisting of all $T \in \mathrm{\Gamma}(1)$ for which $T \equiv \pm I(\bmod N)$, where $N$ is a positive integer and $I$ is the identity matrix. A subgroup $\mathscr{G}$ of $\widehat{\Gamma}(1)$ is said to be a congruence group of level $N$ if $\mathscr{G}$ contains $\Gamma(N)$ and $N$ is the least such integer. Similarly, we denote by $\Gamma(N)$ the principal congruence subgroup of level $N$ of $\Gamma(1)$, consisting of those $T \in \Gamma(1)$ for which $T \equiv I(\bmod N)$, and we say that a subgroup $\mathscr{G}$ of $\Gamma(1)$ is a congruence group of level $N$ if $\mathscr{G}$ contains $\Gamma(N)$ and $N$ is minimal with respect to this property. In a recent paper [9] Rankin considered lattice subgroups of a free congruence subgroup $\hat{F}_{n}$ of rank $n$ of $\hat{\Gamma}(1)$. By a lattice subgroup of $\hat{F}_{n}$ we mean a subgroup of $\hat{F}_{n}$ which contains the commutator group $\hat{F}_{n}^{\prime}$. In particular, he showed that, if $\hat{F}_{n}$ is a congruence group of level $N$ and if $\mathscr{G}$ is a lattice congruence subgroup of $\hat{F}_{n}$ of level $q r$, where $r$ is the largest divisor of $q r$ prime to $N$, then $N$ divides $q$ and $r$ divides 12 . He then posed the problem of finding an upper bound for the factor $q$. It is the purpose of this paper to find such an upper bound for $q$. We also consider bounds for the factor $r$.

We note that, if $\mathscr{G}$ is a lattice congruence subgroup of level $q r$ of a free congruence subgroup $\hat{F}_{n}$ of level $N$, then $\cap \hat{G}(N)$ is a lattice congruence subgroup of $\hat{\Gamma}(N)$ of level gr. This reduces the problem to the consideration of lattice subgroups of $\hat{\Gamma}(N)$ which are congruence groups of level $q r$. We may also assume that such a lattice subgroup is normal in $\hat{\Gamma}(1)$, since the intersection of its conjugates is also a lattice congruence subgroup of $f(N)$ of the same level $q r$. We therefore confine our attention to lattice subgroups of $\Gamma(N)$ in $\Gamma(1)$ which are congruence groups of level $q r$ and which are normal in $\Gamma(1)$. We use McQuillan's classification of normal congruence subgroups of $\Gamma(1)[4]$ and follow his notation.

2. Let $G \cong \prod_{i=1}^{s} G_{i}$, the direct product of $s$ finite groups $G_{i}$, and let $L$ be a subgroup of $G$. We let $F_{i}=\left\{g_{i} \in G_{i}:\left(1,1, \ldots, g_{i}, \ldots, 1\right) \in L\right\}$ and call $F_{i}$ the $i$ th foot of $L$.

THEOREM 1. If $L$ is a normal subgroup of $G$, then $F_{i}$ is normal in $G_{i}$. If, in addition, $G / L$ is abelian, then $G_{i} / F_{i}$ is abelian $(1 \leqq i \leqq s)$.

The proof is clear and is omitted.

Now let $N=\prod_{i=1}^{t} p_{i}^{\alpha_{i}}$ and $q=\prod_{i=1}^{t} p_{i}^{\beta_{i}}$, where $p_{i}$ is prime and $\beta_{i} \geqq \alpha_{i}>0(1 \leqq i \leqq t)$.

THEOREM 2. $\Gamma(N) / \Gamma(q r) \cong \Gamma(1) / \Gamma(r) \times \prod_{i=1}^{t} \Gamma\left(p_{i}^{\alpha_{i}}\right) / \Gamma\left(p_{i}^{\beta_{i}}\right)$. 
Proof. It is well known [7] that $\Gamma(d) / \Gamma(d m n) \cong \Gamma(d) / \Gamma(d m) \times \Gamma(d) / \Gamma(d n)$, when $d$ is an arbitrary positive integer and $(m, n)=1$. By repeated applications of the above we obtain

$$
\Gamma(N) / \Gamma(q r) \cong \Gamma(N) / \Gamma(N r) \times \prod_{i=1}^{r} \Gamma(N) / \Gamma\left(N p_{i}^{\beta_{i}-\alpha_{i}}\right) .
$$

The result follows since $(r, N)=1$, and so

$$
\Gamma(N) / \Gamma(N r) \cong \Gamma(1) / \Gamma(r)
$$

Also

$$
\Gamma(N) / \Gamma\left(N p_{i}^{\beta_{i}-\alpha_{i}}\right) \cong \Gamma\left(p_{i}^{\alpha_{i}}\right) / \Gamma\left(p_{i}^{\beta_{i}}\right) \quad(1 \leqq i \leqq t)
$$

We write

$$
I=\left[\begin{array}{ll}
1 & 0 \\
0 & 1
\end{array}\right], \quad U=\left[\begin{array}{ll}
1 & 1 \\
0 & 1
\end{array}\right], \quad W=\left[\begin{array}{ll}
1 & 0 \\
1 & 1
\end{array}\right] .
$$

Let $\mathscr{G}^{*}$ be a lattice subgroup of $\Gamma(N)$ and let it be a congruence group of level $q r$. As previously stated, we may take $\mathscr{G}^{*}$ to be normal in $\Gamma(1)$. Let

$$
\mathscr{G} \cong \mathscr{G}^{*} / \Gamma(q r)
$$

Denote the foot of $\mathscr{G}$ in $\Gamma(1) / \Gamma(r)$ and $\Gamma\left(p_{i}^{\alpha_{i}}\right) / \Gamma\left(p_{i}^{\beta_{l}}\right)$ by $F$ and $F_{i}$ respectively $(1 \leqq i \leqq t)$. We let

$$
F \cong F^{*} / \Gamma(r) \quad \text { and } \quad F_{i} \cong F_{i}^{*} / \Gamma\left(p_{i}^{\beta_{i}}\right)
$$

We now apply Theorem 1 (with $L=\mathscr{G}$ ) and conclude that $F^{*}$ is a normal congruence subgroup of $\Gamma(1)$ of level $r$, such that $\Gamma(1) / F^{*}$ is abelian, and that $F_{i}^{*}$ is a normal congruence subgroup of $\Gamma(1)$ of level $p_{i}^{\beta_{i}}$, such that $\Gamma\left(p_{i}^{\alpha}\right) / F_{i}^{*}$ is abelian.

THEOREM 3. The factor $r$ divides 12 .

Proof. Clearly $F^{*} \supseteq \Gamma^{\prime}(1)$, where $\Gamma^{\prime}(1)$ is the commutator subgroup of $\Gamma(1)$. The result follows since van Lint has shown [3] that $\Gamma^{\prime}(1)$ is a congruence group of level 12.

THEOREM 4. If $p_{i}$ is an odd prime and $F_{i}^{*}$ is a lattice subgroup of $\Gamma\left(p_{i}^{\alpha_{i}}\right)$ and a normal congruence subgroup of $\Gamma(1)$ of level $p_{i}^{\beta_{t}}$, then $p_{i}^{\beta_{t}}$ divides $p_{i}^{2 \alpha_{t}}$.

Proof. For $p_{i}$ odd, Section 3 of McQuillan's paper shows that $F_{i}^{*}$ is either $\Gamma\left(p_{i}^{\beta_{t}}\right)$ or $\Gamma\left(p_{i}^{\beta_{i}}\right)$, when $\Gamma(l)=\{A \in \Gamma(1): A \equiv \pm I(\bmod l)\}$.

Now if $F_{i}^{*}=\bar{\Gamma}\left(p_{i}^{\beta_{i}}\right)$, then $-I \in F_{i}^{*}$. As $F_{i}^{*} \subseteq \Gamma\left(p_{i}^{\alpha_{i}}\right)$, this implies that $-I \equiv I\left(\bmod p_{l}^{\alpha_{i}}\right)$, which leads to a contradiction as $p_{i}$ is odd and $\alpha_{i}>0$. 
Hence $F_{i}^{*}=\Gamma\left(p_{i}^{\beta_{i}}\right)$, and we have that $\Gamma\left(p_{i}^{\alpha_{i}}\right) / \Gamma\left(p_{i}^{\beta_{1}}\right)$ is abelian. Thus we must have

$$
U^{p_{i}^{\alpha} i} W^{p_{i}^{\alpha} i} \equiv W^{p_{i}^{\alpha} i} U^{p_{i}^{\alpha} i} \quad\left(\bmod p_{i}^{\beta_{i}}\right)
$$

which is true if and only if $p_{i}^{\beta_{t}}$ divides $p_{i}^{2 \alpha_{t}}$.

We now consider lattice subgroups of $\Gamma\left(2^{n}\right)$ which are normal congruence subgroups of $\Gamma(1)$ of level $2^{m}$. Let $G^{*}$ be such a subgroup and set

$$
\mathscr{G} \cong \mathscr{G}^{*} / \Gamma\left(2^{m}\right)
$$

The non-trivial possibilities for $\mathscr{G}$ are, in McQuillan's notation,

$$
\mathscr{G}=Z\left(2^{m}\right) ; \quad E_{m}, \pm E_{m}(m \geqq 2) ; \quad C_{m}, H_{m}, \Lambda_{m}(m \geqq 3) ; \quad D_{m}, F_{m}, \pm D_{m}(m \geqq 4) .
$$

The group $\Lambda_{m}$ is not listed by McQuillan but is the normal subgroup corresponding to $\Gamma\left(2^{m}\right)$, i.e. $\Lambda_{m} \cong \bar{\Gamma}\left(2^{m}\right) / \Gamma\left(2^{m}\right)$. $\boldsymbol{K}_{m}^{u}$.

We consider the cases $n=1$ and $n>1$ separately. We denote the group $\Gamma\left(2^{u}\right) / \Gamma\left(2^{m}\right)$ by

THEOREM 5. If $\mathscr{G}^{*}$ is a lattice subgroup of $\Gamma(2)$ and is a normal congruence subgroup of $\Gamma(1)$ of level $2^{m}$, then $2^{m}$ divides 16 .

Proof. The non-trivial possibilities for $\mathscr{G}$ are as listed above. We note that two matrices $A, B \in \mathscr{G}$ are considered as equal if and only if $A \equiv B\left(\bmod 2^{m}\right)$.

Now it is readily seen that all the possible groups $\mathscr{G}$ have elements whose $(1,2)$ and $(2,1)$ entries are either zero or $2^{m-1}$. Now, considering $U^{2}$ and $W^{2}$ as members of $K_{m}^{1}$, we have, since $K_{m}^{1} / \mathscr{G}$ is abelian,

$$
\left[U^{2}, W^{2}\right]=U^{2} W^{2} U^{-2} W^{-2} \in \mathscr{G} .
$$

As $\left[U^{2}, W^{2}\right]=\left[\begin{array}{rr}21 & -8 \\ 8 & -3\end{array}\right]$, this implies that $8 \equiv 0$ or $2^{m-1}\left(\bmod 2^{m}\right)$. These two congruences combine into $16 \equiv 0\left(\bmod 2^{m}\right)$, which gives the required result.

Now

$$
D_{4}=\left\{\left[\begin{array}{ll}
1 & 0 \\
0 & 1
\end{array}\right],\left[\begin{array}{rr}
5 & 8 \\
8 & -3
\end{array}\right],\left[\begin{array}{ll}
9 & 0 \\
0 & 9
\end{array}\right],\left[\begin{array}{rr}
-3 & 8 \\
8 & 5
\end{array}\right]\right\}
$$

when the matrices are considered modulo 16 . Let

$$
D_{4} \cong D_{4}^{*} / \Gamma(16) \text {. }
$$

$\Gamma(2)$ is generated by $\pm U^{2}, W^{2}$, and hence the commutator subgroup $\Gamma^{\prime}(2)$ is generated by $\left[U^{2 \lambda}, W^{2 \mu}\right]$, with $\lambda, \mu$ integral. Since

$$
\left[U^{2 \lambda}, W^{2 \mu}\right] \equiv\left[\begin{array}{cc}
1+4 \lambda \mu & -8 \mu \lambda^{2} \\
8 \mu^{2} \lambda & 1-4 \lambda \mu
\end{array}\right] \quad(\bmod 16)
$$


it can be readily verified that $\left[U^{2 \lambda}, W^{2 \mu}\right] \in D_{4}^{*}$, for all $\lambda, \mu$. Hence $D_{4}^{*}$ is a lattice subgroup of $\Gamma(2)$ of level 16, which shows that the upper bound for $2^{m}$ is in fact attained. We note finally that $\pm D_{4}^{*}$ is also a lattice subgroup of $\Gamma(2)$ of level 16 , where

$$
\pm D_{4} \cong \pm D_{4}^{*} / \Gamma(16)
$$

We now assume that $n>1$ and consider the non-trivial possibilities for the group $\mathscr{G}$, listed previously.

LEMMA 1. $\mathscr{G} \neq \Lambda_{m}, \pm E_{m}, \pm D_{m}, Z\left(2^{m}\right)$.

Proof. The result follows because each of the above groups corresponds to a subgroup of $\Gamma(1)$ containing $-I$.

Now $\mathscr{G} \subseteq K_{m}^{n}$ and $-I \notin \Gamma\left(2^{n}\right)$, when $n>1$. We shall assume from now on without loss of generality that $m>n$.

LEMMA 2. If $m>n, \mathscr{G} \neq H_{m}$ and $\mathscr{G} \neq F_{m}$.

Proof. If $\mathscr{G}=H_{m}$, we have, since $\mathscr{G} \subseteq K_{m}^{n}$,

$$
\left[\begin{array}{cc}
-1+2^{m-1} & 0 \\
0 & -1+2^{m-1}
\end{array}\right] \equiv I \quad\left(\bmod 2^{n}\right)
$$

which is untrue as $n>1$. Thus $\mathscr{G} \neq H_{m}$.

If $\mathscr{G}=F_{m}$, we have, since $\mathscr{G} \subseteq K_{m}^{n}$,

$$
\left[\begin{array}{cc}
-1-2^{m-2} & 0 \\
0 & -1+2^{m-2}
\end{array}\right] \equiv I \quad\left(\bmod 2^{n}\right) .
$$

This yields the congruence $2^{m-2} \equiv 2\left(\bmod 2^{n}\right)$.

Now we are assuming that $m-2 \geqq n-1$. If $m-2>n-1$, then the congruence reduces to $2 \equiv 0\left(\bmod 2^{n}\right)$, which is not so. If $m-2=n-1$, then we have $2^{n-1} \equiv 2\left(\bmod 2^{n}\right)$, which is only true if $n=2$. But the existence of the group $F_{m}$ ensures that $m \geqq 4$. Hence $n+1 \geqq 4$ and so $n \geqq 3$, which gives the required contradiction.

Thus we may conclude that $\mathscr{G} \neq F_{m}$.

We shall now use Lemmas 1 and 2 to obtain the following theorem.

THEOREM 6. If $\mathscr{G}^{*}$ is a lattice subgroup of $\Gamma\left(2^{n}\right)$ and is a normal congruence subgroup of $\Gamma(1)$ of level $2^{m}$, then $2^{m}$ divides $2^{2 n+1}$.

Proof. Lemmas 1 and 2 show that the remaining possibilities for $\mathscr{G}$ are

$$
\mathscr{G}=D_{m}, E_{m}, C_{m}
$$

Suppose now that $\mathscr{G}=D_{m}$; we make the further assumption, again without loss of generality, that $m>n+1$. Now $D_{m} \subseteq K_{m}^{m-2} \subseteq K_{m}^{n}$, if $m>n+1$. But we know that $K_{m}^{n} / D_{m}$ is 
abelian. Hence $K_{m}^{m-2} / D_{m}$ is abelian and

$$
\left(K_{m}^{n} / D_{m}\right) /\left(K_{m}^{m-2} / D_{m}\right) \cong K_{m}^{n} / K_{m}^{m-2} \cong \Gamma\left(2^{n}\right) / \Gamma\left(2^{m-2}\right) .
$$

Thus $\Gamma\left(2^{n}\right) / \Gamma\left(2^{m-2}\right)$ is abelian and so

$$
U^{2^{n}} W^{2^{n}} \equiv W^{2^{n}} U^{2^{n}} \quad\left(\bmod 2^{m-2}\right),
$$

which is true if and only if $2^{m}$ divides $2^{2 n+2}$.

However, if $m=2 n+2$, then the fact that $K_{2 n+2}^{n} / D_{2 n+2}$ is abelian implies that

$$
\left[U^{2^{n}}, W^{2^{n}}\right] \in D_{2 n+2} \text {. }
$$

Now

$$
\left[U^{2 n}, W^{2 n}\right] \equiv\left[\begin{array}{cc}
1+2^{2 n} & 0 \\
0 & 1-2^{2 n}
\end{array}\right] \quad\left(\bmod 2^{2 n+2}\right) .
$$

A simple inspection shows that $\left[U^{2 n}, W^{2 n}\right] \notin D_{2 n+2}$. Hence we conclude that, if $\mathscr{G}=D_{m}$, then $2^{m}$ divides $2^{2 n+1}$.

Suppose now that $\mathscr{G}=E_{m}$ or $C_{m}$. Now both $E_{m}$ and $C_{m} \subseteq K_{m}^{m-1} \subseteq K_{m}^{n}$, for $m>n$. Using an argument similar to that given in the first part of the theorem, we can show that the fact that $K_{m}^{n} / \mathscr{G}$ is abelian implies that $\Gamma\left(2^{n}\right) / \Gamma\left(2^{m-1}\right)$ is abelian, for $\mathscr{G}=E_{m}$ or $C_{m}$. Thus

$$
\left[U^{2^{n}}, W^{2^{n}}\right] \equiv I \quad\left(\bmod 2^{m-1}\right)
$$

which is true if and only if $2^{m}$ divides $2^{2 n+1}$.

The theorem is thus established and we now show that the upper bound of $2^{2 n+1}$ is attained. Let

$$
C_{2 n+1} \cong C_{2 n+1}^{*} / \Gamma\left(2^{2 n+1}\right) .
$$

and suppose that $A, B \in \Gamma\left(2^{n}\right)$. Then

and

$$
A=\left[\begin{array}{cc}
1+a 2^{n} & b 2^{n} \\
c 2^{n} & 1+d 2^{n}
\end{array}\right], \quad \text { where }(a+d)=2^{n}(b c-a d)
$$

$$
B=\left[\begin{array}{cc}
1+e 2^{n} & f 2^{n} \\
g 2^{n} & 1+h 2^{n}
\end{array}\right], \quad \text { where }(e+h)=2^{n}(f g-e h) .
$$

Thus

and

$$
A B=\left[\begin{array}{cc}
1+(a+e) 2^{n}+(a e+b g) 2^{2 n} & (b+f) 2^{n}+(a f+b h) 2^{2 n} \\
(c+g) 2^{n}+(c e+g d) 2^{2 n} & 1+(h+d) 2^{n}+(c f+h d) 2^{2 n}
\end{array}\right],
$$

$$
A^{-1} B^{-1}=\left[\begin{array}{cc}
1+(h+d) 2^{n}+(h d+b g) 2^{2 n} & -\left\{(b+f) 2^{n}+(f d+b e) 2^{2 n}\right\} \\
-\left\{(c+g) 2^{n}+(h c+a g) 2^{2 n}\right\} & 1+(a+e) 2^{n}+(a e+f c) 2^{2 n}
\end{array}\right] .
$$


Now

$$
[A, B]=A B A^{-1} B^{-1} \equiv\left[\begin{array}{cc}
1+(b g-f c) 2^{2 n} & 0 \\
0 & 1+(f c-b g) 2^{2 n}
\end{array}\right] \quad\left(\bmod 2^{2 n+1}\right) .
$$

Thus

$$
[A, B] \equiv I \text { or }\left[\begin{array}{cc}
1+2^{2 n} & 0 \\
0 & 1+2^{2 n}
\end{array}\right] \quad\left(\bmod 2^{2 n+1}\right) .
$$

This implies that $[A, B] \in C_{2 n+1}^{*}$, for all $A, B \in \Gamma\left(2^{n}\right)$. Thus $C_{2 n+1}^{*}$ is a lattice subgroup of $\Gamma\left(2^{n}\right)$ and is a congruence group whose level is equal to the upper bound of $2^{2 n+1}$.

We now tabulate the results, quoting the upper bounds for the factors $q$ and $r$ in each case and stating a lattice subgroup of $\Gamma(N)$ which is a congruence group whose level is equal to the upper bound for $q r$, denoted by $\max q r$.

We shall make use of the groups $\Gamma^{\prime}(1), M^{*}$ and $\Gamma^{4}$. The group $M^{*}$ is the group corresponding to $M$ quoted by McQuillan. $M^{*}$ is a normal congruence subgroup of $\Gamma(1)$ of level 3 containing $\Gamma^{\prime}(1)$. The group $\Gamma^{4}$ is not listed by McQuillan. It is the subgroup of $\Gamma(1)$ generated by $\left[\begin{array}{ll}0 & -1 \\ 1 & -1\end{array}\right]$ and $\left[\begin{array}{rr}-1 & -1 \\ 1 & 0\end{array}\right]$ and can readily be shown to be a normal congruence subgroup of $\Gamma(1)$ of level 4 , containing $\Gamma^{\prime}(1)$. We put $N=2^{n} l$, where $n \geqq 0$ and $l$ is odd.

TABLE

\begin{tabular}{c|c|c|c|l}
\hline$(N, 12)$ & $q$ divides & $r$ divides & $\max g r$ & $\max q r$ attained by \\
\cline { 1 - 3 } & $N^{2}$ & 12 & $12 N^{2}$ & $\Gamma^{\prime}(1) \cap \Gamma\left(N^{2}\right)$ \\
2 & $4 N^{2}$ & 3 & $12 N^{2}$ & $D_{4}^{*} \cap \Gamma\left(l^{2}\right) \cap M^{*}$ \\
3 & $N^{2}$ & 4 & $4 N^{2}$ & $\Gamma\left(N^{2}\right) \cap \Gamma^{4}$ \\
4 & $2 N^{2}$ & 3 & $6 N^{2}$ & $C_{2 n+1}^{*} \cap \Gamma\left(l^{2}\right) \cap M^{*}$ \\
6 & $4 N^{2}$ & 1 & $4 N^{2}$ & $D_{4}^{*} \cap \Gamma\left(l^{2}\right)$ \\
12 & $2 N^{2}$ & 1 & $2 N^{2}$ & $C_{2 n+1}^{*} \cap \Gamma\left(l^{2}\right)$ \\
& & & & \\
\hline
\end{tabular}

Further, for all integers $d$ such that $N$ divides $d$ and $d$ divides max $q r$, a lattice subgroup of $\Gamma(N)$ which is a congruence group of level $d$ may be readily produced.

3. We now extend the results of the table to the inhomogeneous modular group $\hat{\Gamma}(1)$.

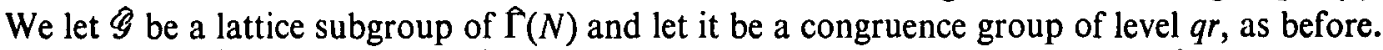
We may take $\mathscr{G}$ to be normal in $\Gamma(1)$. Let $\bar{G}$ be the corresponding subgroup to $\bar{G}$ in $\Gamma$ (1). We have $\cong \mathscr{G} / \Lambda$, where $\Lambda=\{I,-I\}$. It is easily verified that $\operatorname{G}$ is a congruence group of level $d$ in $\mathrm{\Gamma}(1)$ if and only if $\bar{G}$ is a congruence group of level $d$ in $\Gamma(1)$.

We consider the cases $N=2$ and $N>2$ separately. 
THEOREM 7. If $\hat{G}$ is a lattice subgroup of $\hat{\Gamma}(2)$ and is a normal congruence subgroup of $\hat{\Gamma}(1)$ of level qr, then qr divides 48.

Proof. We have $\Gamma(2) \cong \Gamma(2) / \Lambda$ and $\mathscr{G} \cong \mathscr{G} / \Lambda$, when $\overline{\mathscr{G}}$ is the group corresponding to $\mathscr{G}$ in $\Gamma(1)$. Then $\bar{G}$ is a normal congruence subgroup of $\Gamma(1)$ and has level $q r$. We also have

$$
\Gamma(2) / \mathscr{G} \cong \Gamma(2) / \overline{\mathscr{G}} \text {. }
$$

Thus, since $\Gamma(2) / \widehat{G}$ is abelian, $\overline{\mathscr{G}}$ is a lattice subgroup of $\Gamma(2)$. The result follows by applying the results of the table for the case $N=2$.

Let $Z^{*}(8), \pm \hat{D}_{4}^{*}$ and $\hat{M}^{*}$ be the subgroups of $\hat{\Gamma}(1)$ corresponding to the subgroups $Z^{*}(8), \pm D_{4}^{*}$ and $M^{*}$ of $\Gamma(1)$ respectively. $Z^{*}(8)$ and $\pm \hat{D}_{4}^{*}$ are lattice congruence subgroups of $\hat{\Gamma}(2)$ of levels 8 and 16 respectively and $\hat{M}^{*}$ is a congruence group of level 3 containing $\hat{\Gamma}^{\prime}(1)$, the commutator subgroup of $\hat{\Gamma}(1)$. The groups $\hat{H} \cap \hat{K}$, where $\hat{H}=\hat{\Gamma}(2), \hat{\Gamma}(4), Z^{*}(8)$ or $\pm \hat{D}_{4}^{*}$, and $\hat{R}=\hat{\Gamma}(1)$ or $\hat{M}^{*}$, form a set of lattice congruence subgroups of $\hat{\Gamma}(2)$, whose levels are equal to the eight even divisors of 48 , including 48 itself.

THEOREM 8. If $N>2$ and $\mathscr{G}$ is a lattice subgroup of $\Gamma(N)$ and is a normal congruence subgroup of $\Gamma(1)$ of level qr, then there exists a lattice subgroup $\mathscr{G}$ of $\Gamma(N)$ in $\Gamma(1)$ such that $\mathscr{G}$ is a normal congruence subgroup of $\Gamma(1)$ of level qr and $\mathscr{G} \cong \mathscr{G}$.

Proof. Let $\overline{\mathscr{G}}$ be defined as before, so that $\hat{\mathscr{G}} \cong \overline{\mathscr{G}} / \Lambda$. We also have

$$
\hat{\Gamma}(N) \cong \bar{\Gamma}(N) / \Lambda \cong \Gamma(N)
$$

Now $\bar{G} \subseteq \bar{\Gamma}(N)$ so that, for any $A \in \bar{G}, A \equiv \pm I(\bmod N)$. We note that, as $N>2$, we cannot have a member of $\bar{G}$ congruent to both $I$ and $-I(\bmod N)$.

We define a subset $\mathscr{G}$ of $\bar{G}$ as follows.

$$
\mathscr{G}=\{A \in \overline{\mathscr{G}}: A \equiv I(\bmod N)\} .
$$

Clearly $\mathscr{G}$ is a subgroup of $\overline{\mathscr{G}}$ contained in $\Gamma(N)$, such that $\mathscr{G} \cong \overline{\mathscr{G}} / \Lambda$. Now $\hat{\Gamma}(q r) \subseteq \mathscr{G}$ and $q r$ is minimal. This implies that $\bar{\Gamma}(q r)$ and hence $\Gamma(q r)$ is contained in $\overline{\mathscr{G}}$.

In fact $\Gamma(q r) \subseteq \mathscr{G}$; for, if not, there exists $X \in \bar{G}$ such that

$$
X \equiv I(\bmod q r) \quad \text { and } \quad X \equiv-I(\bmod N) .
$$

This yields a contradiction, since $N$ divides $q r$ and $N>2$. Moreover the level of $\mathscr{G}$ is exactly $q r$; for if there exists $d<q r$ such that $\Gamma(d) \subseteq \mathscr{G}$, then $\Gamma(d) \subseteq \mathscr{G}$, which contradicts the minimality of $q r$.

Finally $\mathscr{G}$ is of course normal in $\Gamma(1)$ and is a lattice subgroup of $\Gamma(N)$; for we have

$$
\hat{I}(N) / \hat{G} \cong \Gamma(N) / \mathscr{G}
$$

We note that, if $\mathscr{G}$ is a lattice subgroup of $\Gamma(N)$, where $N>2$, and $\mathscr{G}$ is a normal congruence subgroup of $\Gamma(1)$ of level $q r$, then the subgroup $\mathscr{G}$ of $\Gamma(1)$ corresponding to $\mathscr{G}$, where $\mathscr{G} \cong \mathscr{G}$, 
is a lattice subgroup of $\hat{\Gamma}(N)$ and is a normal congruence subgroup of $\hat{\Gamma}(1)$ of level $q r$. The fact that its level is exactly $q r$ follows from Theorem 8 . For, clearly, $\Gamma(q r) \subseteq \mathscr{G}$ and, if $\Gamma(d) \subseteq \mathscr{G}$ with $d<q r$, then Theorem 8 shows that $\Gamma(d) \subseteq \mathscr{G}$, which contradicts the minimality of $q r$.

Theorems 7 and 8 show that the upper bounds for $q r$ in $\Gamma(1)$, shown in the table, also hold in $\hat{\Gamma}(1)$. Moreover the remarks following the two theorems show that the upper bounds are attained in $\mathrm{f}(1)$ in all cases. More generally, it is easily seen that, for all integers $d$ such that $d$ divides max $q r$ and $N$ divides $d$, there exists a lattice subgroup of $\hat{\Gamma}(N)$ which is a congruence group of level $d$. In particular we note that in $\Gamma(1)$ the factor $r$ divides 12 in all cases, a result obtained by Rankin by different methods.

4. In this section we introduce an infinite class of lattice subgroups of $\hat{\Gamma}(N)$ and use the results of the previous section to investigate which of these groups is a congruence group. These subgroups provide a natural extension to the subgroups $\Omega(p, S)$ of $\Gamma(p)$ ( $p$ a prime) introduced by Reiner [10], and for $p=2$ by Fricke [1] and Pick [8], and contain an infinite set of subgroups of finite index in $\uparrow(1)$ which are not congruence groups.

Lemma 3. For $N>1, U^{N}$ may be taken as a free generator of $\hat{\Gamma}(N)$.

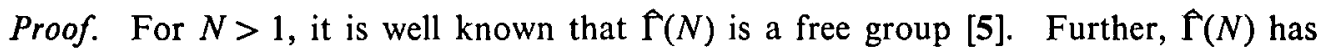
$n=\mu / N>1$ incongruent cusps, where $\mu=[\hat{\Gamma}(1): \hat{\Gamma}(N)]$. Now, by Section VI, 4 (p. 241) of [2], $\hat{\Gamma}(N)$ has a canonical fundamental region with $n$ incongruent parabolic vertices and $4 g+n$ other " accidental" vertices, which are all congruent to each other $\bmod \hat{\Gamma}(N)$, where $g$ is the genus of $\mathrm{\Gamma}(N)$. The $n$ parabolic vertices determine $n$ parabolic generators $P_{1}, \ldots, P_{n}$, which form part of a set of $2 g+n$ generators of $\hat{\Gamma}(N)$, and which satisfy one relation only (Theorem, p. 234 of [2]). We may take $P_{1}=U^{N}$, and, as $n>1$, we may use the relation to eliminate $P_{2}$, leaving a set of free generators of $\hat{\Gamma}(N)$, one of which is $U^{N}$.

LEMmA 4. The rank of $\hat{\Gamma}(2)$ is 2 and, when $N>2$, the rank of $\hat{\Gamma}(N)$ is $1+\mu(N) / 12$, where

$$
\mu(N)=N^{3} \prod_{p / N}\left(1-\frac{1}{p^{2}}\right) .
$$

Proof. It is known that $\Gamma(2)$ is freely generated by $U^{2}$ and $W^{2}$. Using this fact we may compute the rank of $\hat{\Upsilon}(2 N)(N>1)$ by the well known Reidemeister-Schreier formula for the rank of a subgroup of finite index in a free group of finite rank. The rank of $\Gamma(N)(N>2)$ may then be calculated from the rank of $\uparrow(2 N)$ using the Reidemeister-Schreier formula in reverse.

Let $P=\uparrow(N) / \hat{\Gamma}^{\prime}(N)$ and let $P^{S}$ be the subgroup of $P$ generated by the $S$ th powers of the elements of $P(S \geqq 1)$. Denote by $\Omega(N, S)$ the inverse image of $P^{S}$ under the canonical mapping of $\Gamma(N)$ onto $P$. It is readily seen that $\Omega(N, S)$ consists of those elements of $\Gamma(N)$ for which the exponent sums with respect to each of the free generators of $\Gamma(N)$ is a multiple of $S$. Clearly $\Omega(N, S)$ is a lattice subgroup of $\Gamma(N)$ and is normal in $\hat{\Gamma}(1)$. It also follows that $[\hat{\Gamma}(N): \Omega(N, S)]=S^{\sigma}$, where $\sigma$ is the rank of $\hat{\Gamma}(N)$. 
Lemma 5. If $S_{1}$ divides $S_{2}$, then $\Omega\left(N, S_{1}\right) \supseteq \Omega\left(N, S_{2}\right)$.

The proof is obvious.

THEOREM 9. $\Omega(N, S)$ is a congruence group if and only if $\Omega(N, S) \supseteq \widehat{\Gamma}(N S)$.

Proof. The level of $\Omega(N, S)$, as defined by Wohlfahrt in [11], is $N S$. This follows from the normality of $\Omega(N, S)$ in $\hat{\Gamma}(1)$ and from the fact that $U^{N}$ may be taken as a free generator of $\hat{\Gamma}(N)$. We obtain the required result by applying Theorem 2 of Wohlfahrt's paper.

We now use Theorem 9 to investigate which of the groups $\Omega(N, S)$ is a congruence group. The results obtained will include these of Reiner, for clearly, when $N$ is a prime $p$, the groups are the subgroups $\Omega(p, S)$ introduced by him. We treat the cases $N=2, N=3$ and $N \geqq 4$ separately.

THEOREM 10. $\Omega(2, S)$ is a congruence subgroup if and only if $S=1,2,4,8$.

Proof. If $\Omega(2, S)$ is a congruence group, then by Theorem 9 and the results shown in the table in Section 2 we may conclude that $S$ divides 24 .

If $S$ is any divisor of 24 divisible by 3 , then Lemma 5 implies that $\Omega(2,3)$ is a congruence subgroup. However, $[\hat{\Gamma}(2): \Omega(2,3)]=9$ and $[\hat{\Gamma}(2): \hat{\Gamma}(6)]=12$, which yields an immediate contradiction. Hence $\Omega(2,3)$ is not a congruence subgroup.

There remain the cases $S=1,2,4,8$ to consider. We note that $\hat{\Gamma}(2)$ is freely generated by $U^{2}$ and $W^{2}$. The proof for the case $S=8$ is to be found in Theorem 3 of a paper by Newman [6]. In fact Newman shows that $\hat{\Gamma}(16)$ is contained in that lattice subgroup of $\hat{\Gamma}(2)$ whose elements have exponent sums with respect to the generator $U^{2}$ that are multiples of 8 . However, elementary modifications of his proof show that any element of $\uparrow(16)$ also has its exponent sum with respect to the generator $W^{2}$ congruent to zero modulo 8 . Since $\Omega(2,8)$ is a congruence group, so also are $\Omega(2,1), \Omega(2,2)$ and $\Omega(2,4)$, by Lemma 5 . Clearly $\Omega(2,1)=\hat{\Gamma}(2)$ and it is also readily verified that $\Omega(2,2)=\Gamma(4), \Omega(2,4)=Z^{*}(8)$ and $\Omega(2,8)= \pm \hat{D}_{4}^{*}$. This completes the proof of the theorem.

THEOREM 11. $\Omega(3, S)$ is a congruence subgroup if and only if $S=1,3$.

Proof. If $\Omega(3, S)$ is a congruence subgroup, then by Theorem 9 and the results for $N=3$ in the table, we conclude that $S$ divides 12 . If $S$ is an even divisor of 12, then Lemma 5 implies that $\Omega(3,2)$ is a congruence subgroup. But $[\Gamma(3): \Omega(3,2)]=8$ and $[\Gamma(3): \digamma(6)]=6$, which yields an immediate contradiction.

Trivially $\Omega(3,1)=\hat{\Gamma}(3)$ and, since $\hat{\Gamma}(3)$ is freely generated by $\left(U^{-1} W\right)^{-v} U^{3}\left(U^{-1} W\right)^{v}$ $(v=1,2,3)$, it is easily verified that $\hat{\Gamma}(9)=\Omega(3,3)$. This completes the proof of the theorem.

THEOREM 12. If $N \geqq 4, \Omega(N, S)$ is a congruence subgroup if and only if $S=1$.

Proof. If $\Omega(N, S)$ is a congruence subgroup, then, by Theorem 9 and the results of the table in Section 2, $S$ divides $12 N$. Clearly $\Omega(N, 1)=\hat{\Gamma}(N)$ which is a congruence subgroup and, if $S>1$, then Lemma 5 implies that $\Omega(N, p)$ is a congruence subgroup, where $p$ is any prime dividing $S$. Now, since $S$ divides $12 N$, this implies that $\Omega(N, p)$ is a congruence subgroup, where $p$ is any prime dividing $N$, or, when $(N, 2)=1$, that $\Omega(N, 2)$ is a congruence 
subgroup, or, when $(N, 3)=1$, that $\Omega(N, 3)$ is a congruence subgroup. We shall obtain a contradiction in the most general case for $(N, 6)=1$. The remaining cases for $N \geqq 4$ are proved similarly.

When $(N, 6)=1$,

$$
[\hat{\Gamma}(N): \hat{\Gamma}(N p)]=p^{3}, \quad[\hat{\Gamma}(N): \hat{\Gamma}(2 N)]=6, \quad[\hat{\Gamma}(N): \hat{\Gamma}(3 N)]=24 .
$$

Also

$$
[\hat{\Gamma}(N): \Omega(N, p)]=p^{\sigma}, \quad[\hat{\Gamma}(N): \Omega(N, 2)]=2^{\sigma}, \quad[\hat{\Gamma}(N): \Omega(N, 3)]=3^{\sigma} .
$$

Contradictions follow in all cases since the formula established in Lemma 4 shows that $\sigma>3$. The proof of the theorem is complete.

We conclude by observing that we may easily extend Lemma 3 in the following way. If $\mathscr{G}$ is any free normal subgroup of finite index $\mu$ in $\Gamma(1)$, whose level, as defined by Wohlfahrt in [11], is $N$, then we may take $U^{N}$ to be a free generator of $\mathscr{G}$, provided $\mu / N>1$. As the commutator subgroup $\hat{G}^{\prime} \subseteq \mathscr{G}$, the level of $\mathscr{G}^{\prime}$ is a multiple of $N$. If $\mu / N>1$ and $U^{N l} \in \mathscr{G}^{\prime}$, for some integer $l$, this implies a non-trivial relation between the free generators of $\mathscr{G}$, which is an obvious contradiction. Hence, when $\mu / N>1$, the level of $\mathscr{G}^{\prime}$ is infinite. In fact $\mu / N>1$ for all free normal subgroups $\mathscr{G}$ of finite index in $\Gamma(1)$, except for $=\Gamma^{\prime}(1)$, when $\mu=N=6$. This discussion shows that Proposition 1 of [9] only holds for $\hat{G}=\hat{\Gamma}^{\prime}(1)$, where the level of $\mathscr{G}^{\prime}=\hat{\Gamma}^{\prime \prime}(1)$ is 6 .

\section{REFERENCES}

1. R. Fricke, Über die Substitutionsgruppen, welche zu den aus Legendre'schen Integralmodul $k^{2}(\omega)$ gezogenen Wuhrzeln gehören, Math. Ann. 28 (1887), 99-118.

2. Joseph Lehner, Discontinuous groups and automorphic functions, Mathematical Surveys, No. VIII, American Mathematical Society (Providence, R.I., 1964).

3. J. H. van Lint, On the multiplier system of the Riemann-Dedekind function $\eta$, Nederl. Akad. Wetensch. Proc. Ser. A 61 (=Indag. Math. 20) (1958), 522-527.

4. D. L. McQuillan, Classification of normal congruence subgroups of the modular group, Amer. J. Math. 87 (1965), 285-296. (1964), 262-265.

6. M. Newman, On a problem of G. Sansone, Ann. Mat. pura appl. 65 (1964), 27-34. 253-257.

7. M. Newman and J. R. Smart, Modulary groups of $t \times t$ matrices, Duke Math. J. 30 (1963),

8. G. Pick, Ubber gewisse ganzzahlige lineare Substitutionen, welche sich nicht durch algebraische Congruenzen erklären lassen, Math. Ann. 28 (1887), 119-124. 215-221.

9. R. A. Rankin, Lattice subgroups of free congruence groups, Inventiones Math 2 (1967),

10. I. Reiner, Normal subgroups of the unimodular group, Illinois J. Math. 2 (1958), 142-144.

11. K. Wohlfahrt, An extension of F. Klein's level concept, Illinois J. Math. 8 (1964), 529-535.

\section{UNIVRSITY OF GLASGOW}

